\title{
Protective Effects of Edaravone Against Hypoxia-Induced Lethality in Mice
}

\section{Fatemeh Shaki ${ }^{1}$, Mina Mokhtaran ${ }^{1}$, Amir Shamshirian $^{2,3}$, Shahram Eslami ${ }^{1}$, Danial Shamshirian ${ }^{4}$, Mohammad Ali Ebrahimzadeh ${ }^{1 *}$}

1. Department of Medicinal Chemistry, School of Pharmacy and Pharmaceutical Sciences Research Center, Hemoglobinopathy Institute, Mazandaran University of Medical Sciences, Sari, Iran.

2. Department of Medical Laboratory Sciences, Student Research Committee, School of Allied Medical Science, Mazandaran University of Medical Sciences, Sari, Iran.

3. Gastrointestinal Cancer Research Center, Mazandaran University of Medical Sciences, Sari, Iran.

4. Chronic Respiratory Diseases Research Center, National Research Institute of Tuberculosis and Lung Diseases (NRITLD), Shahid Beheshti University of Medical Sciences, Tehran, Iran.

* Corresponding author: Dr. Mohammad Ali Ebrahimzadeh, Department of Medicinal Chemistry, School of Pharmacy and Pharmaceutical Sciences Research Center, Hemoglobinopathy Institute, Mazandaran University of Medical Sciences, Sari, Iran.

Tel. +98 11 33543081-3; Fax: +98 11 33543084, PO Box- 48175/861

E-mail address: zadeh20@gmail.com 


\title{
Protective Effects of Edaravone Against Hypoxia-Induced Lethality in Mice
}

\begin{abstract}
Edaravone is used for the treatment of acute cerebral infarction in Japan. However, nothing is known about the protective effects of this drug against hypoxia-induced lethality. In this study, the protective effects of edaravone against hypoxia-induced lethality and oxidative stress in mice were evaluated by three experimental models of hypoxia, which are asphyctic, haemic, and circulatory. Statistically significant protective activities were established in all tested models of hypoxia. Antihypoxic activities were especially pronounced in asphytic and circulatory hypoxia. The effect was dose-dependent. Edaravone, at $5 \mathrm{mg} \mathrm{kg}^{-1}$, showed statistically significant activities respect to the control groups. It significantly prolonged the latency for death. At $2.5 \mathrm{mg}$ $\mathrm{kg}^{-1}$, it also prolonged survival time $(26.08 \pm 5.26 \mathrm{~min})$, but this effect was not statistically significant from the control $(P>0.05)$. On the other hand, edaravone significantly inhibited hypoxia-induced oxidative stress (lipid peroxidation and glutathione oxidation) in three models of hypoxia. In conclusion, the results obtained in this study showed that Edaravone has very good protective effects against the hypoxia in all tested models.
\end{abstract}

Keywords: Antihypoxia; Asphyctic hypoxia, Edaravone, Oxidative stress. 


\section{Introduction}

The imbalance between the supply of oxygen and its demand determines the organ hypoxia. It occurs mainly in ischemia and heart diseases, leading to numerous deleterious effects in different tissue, especially in the brain ${ }^{1}$. The brain has a high oxygen consumption, which comprises 20$25 \%$ of total body $\mathrm{O}_{2}$ consumption; therefore, it is susceptible to hypoxia.

Several mechanisms have been reported that contribute to the short-term or long-term brain damage after hypoxia or ischemia, and oxidative stress has been considered as a key mechanism that underlies the hypoxic-ischemic brain injury. On the other hand, the brain is very susceptible to harmful effects of oxidative stress because of (i) an abundant content of polyunsaturated fatty acid, (ii) low level of antioxidant system, (iii) high mitochondrial content and therefore high rate of $\mathrm{O}_{2}$ utilization and (iv) high concentration of transition metals such as copper and iron which could facilitate the formation of hydroxyl radical by Fenton reaction ${ }^{2}$.

Reactive oxygen species (ROS) can trigger various signaling pathways, induce oxidation of lipid, protein, DNA, and non-enzyme antioxidant like glutathione (GSH) inside the cell and subsequently leads to membrane disruption and finally cell death ${ }^{3}$. Therefore, antioxidants that can inhibit or prevent oxidative damage and can protect cells from hypoxic damage. Hypoxia causes oxidative stress, which involves the production of ROS ${ }^{4}$. It has proven that the compounds with antioxidant activity may scavenge ROS and able to exhibit antihypoxic properties.

Edaravone, a novel and potent free radical scavenger, has been shown to have protective effects against cerebral ischemia-reperfusion injuries in some experimental animal models ${ }^{5,6}$. It inhibits the activation of lipoxygenase and peroxidation of the phosphatidylcholine liposomal membrane in vitro ${ }^{5}$. The clinical efficacy of edaravone against ischemic brain attack has been demonstrated by the presence of significant improvements in functional outcome in a human study ${ }^{5,6}$. Edaravone has been prescribed clinically in Japan for the treatment of acute brain infarction since 2001, reducing the mortality rate when administered during the acute stage of stroke ${ }^{6,7}$. Several Japanese studies have shown its efficiency in patients with acute ischemic stroke ${ }^{8-10}$. Edaravone was found to reduce apoptosis. It has been shown to inhibit the accumulations of oxidative by-products and cell death after transient focal ischemia in the brain. These protective effects of edaravone are thought to be attributable to its scavenging of ROS ${ }^{6,11}$. Systemic administration of edaravone attenuated the increase of malondialdehyde levels, the reduction of superoxide dismutase activity, and the suppression of retinal dysfunctions after retinal ischemia/reperfusion in rats ${ }^{12}$.

However, there was no data available on the effect of edaravone in hypoxic conditions. Present work aimed to determine the antihypoxic activities of edaravone in order to understand a possible mechanism of its action in cerebrovascular diseases. 


\section{Material and methods}

\subsection{Experimental animals and diet}

Male Swiss albino mice (20 $\pm 2 \mathrm{~g})$ were randomly housed in groups of 10 in polypropylene cages at ambient temperature, $25 \pm 1{ }^{\circ} \mathrm{C}$ and $45-55 \%$ relative humidity, with a $12 \mathrm{~h}$ light: $12 \mathrm{~h}$ dark cycle (lights on at 7 a.m.). The animals had free access to standard pellet and water and libitum. Experiments were conducted between 8:00 and 14:00 h. All the experimental procedures were conducted in accordance with the NIH guidelines of the Laboratory Animal Care and Use. The Institutional Animal Ethical Committee of Mazandaran University of Medical Sciences also approved the experimental protocol.

\subsection{Asphyctic Hypoxia}

The animals were subjected to hypoxia by putting them individually in a tightly closed $300 \mathrm{ml}$ glass container which was placed under water in an aquarium of $25^{\circ} \mathrm{C}$. The animals had convulsions and died from hypoxia. The latencies for death were recorded. The animals died approximately $2 \mathrm{~min}$ following convulsions. Mice received single i.p. injections of 2.5 and $5 \mathrm{mg}$ $\mathrm{kg}^{-1}$ doses of edaravone for 4 consecutive days. At the $4^{\text {th }}$ day, edaravone or phenytoin $(50 \mathrm{mg}$ $\mathrm{kg}^{-1}$ ) was injected $30 \mathrm{~min}$ before they were subjected to hypoxia. Another control group was treated with normal saline at the same way ${ }^{13}$.

\subsection{Haemic Hypoxia}

Forty mice were divided into five groups each containing eight mice. Control group was treated with normal saline. Mice received single i.p. injections of 5 and $10 \mathrm{mg} \mathrm{kg}^{-1}$ doses of edaravone for four consecutive days. At the $4^{\text {th }}$ day, thirty minutes after i.p. administration of edaravone, $\mathrm{NaNO}_{2}$ (360 mg kg-1) was applied i.p. to mice and antihypoxic activity was estimated as the latent time of evidence of hypoxia in minutes ${ }^{14}$.

\subsection{Circulatory Hypoxia}

Forty mice were divided into five groups each containing eight mice. The groups were treated with normal saline. Mice received single i.p. injections of 2.5 and $5 \mathrm{mg} \mathrm{kg}^{-1}$ doses of edaravone for four consecutive days. At the $4^{\text {th }}$ day, thirty minutes after i.p. administration of edaravone, $\mathrm{NaF}$ (150 mg kg-1) was applied i.p. to mice and antihypoxic activity was estimated in minutes as the latent time of evidence of hypoxia ${ }^{14}$.

\subsection{Oxidative stress assessment:}

After induction of hypoxia via three models, evaluation and comparison the role and severity of oxidative stress between control group and rat receiving different dose of edaravone were performed. After a cut off time in each three models of hypoxia, animal in each group were killed and their brain tissues were isolated for assessment of LPO and GSH. 


\subsection{Measurement of Lipid peroxidation (LPO)}

The content of malondialdehyde (MDA) was determined by using the method of Zhang at al. 2008. $0.25 \mathrm{ml}$ phosphoric acid $(0.05 \mathrm{M})$ was added to $0.2 \mathrm{ml}$ of brain tissue homogenate with the addition of $0.3 \mathrm{ml} 0.2 \%$ thiobarbituric acid. All the samples were placed in a boiling water bath for $30 \mathrm{~min}$. At the end, the tubes were shifted to an ice-bath and $0.4 \mathrm{ml} \mathrm{n}$-butanol was added to each tube. Then, they were centrifuged at $3500 \mathrm{rpm}$ for $10 \mathrm{~min}$. The amount of MDA formed in each of the samples was assessed through measuring the absorbance of the supernatant at $532 \mathrm{~nm}$ with an ELISA reader (ELX800, Biotek, USA). Tetramethoxypropane was used as standard and MDA content was expressed as $\mathrm{nmol} / \mathrm{mg}$ protein ${ }^{15}$.

\subsection{Measurement of glutathione content}

Glutathione (GSH) content was determined by DTNB as an indicator and spectrophotometer. Brifely $0.1 \mathrm{ml}$ of brain tissue was added into $0.1 \mathrm{~mol} / \mathrm{l}$ of phosphate buffers and $0.04 \%$ DTNB in a total volume of $3.0 \mathrm{ml}(\mathrm{pH} \mathrm{7.4)}$. Then developed yellow color, was read at $412 \mathrm{~nm}$ on a spectrophotometer (CE2501, CECIL, France) GSH content was expressed as $\mu \mathrm{g} / \mathrm{mg}$ protein ${ }^{16}$.

\subsection{Statistical Analysis}

Data were presented as mean \pm SD. Analysis of variance (ANOVA) was performed. Duncan's new multiple-range test was used to determine the differences in means. All $p$ values less than 0.05 were regarded as significant. 


\section{Results}

The results of asphytic hypoxia are shown in Figure 1. The results showed that the effect was dose-dependent. Edaravone, at $5 \mathrm{mg} \mathrm{kg}^{-1}$, showed statistically significant activity respect to the control group $(P<0.01)$. It significantly prolonged the latency for death concerning the control group $(33.10 \pm 9.04$ vs. $21.25 \pm 3.83 \mathrm{~min}, P<0.01)$. At $2.5 \mathrm{mg} \mathrm{kg}^{-1}$, it also prolonged survival time $(26.08 \pm 5.26 \mathrm{~min})$, but this effect was not statistically significant from the control $(P>0.05)$.

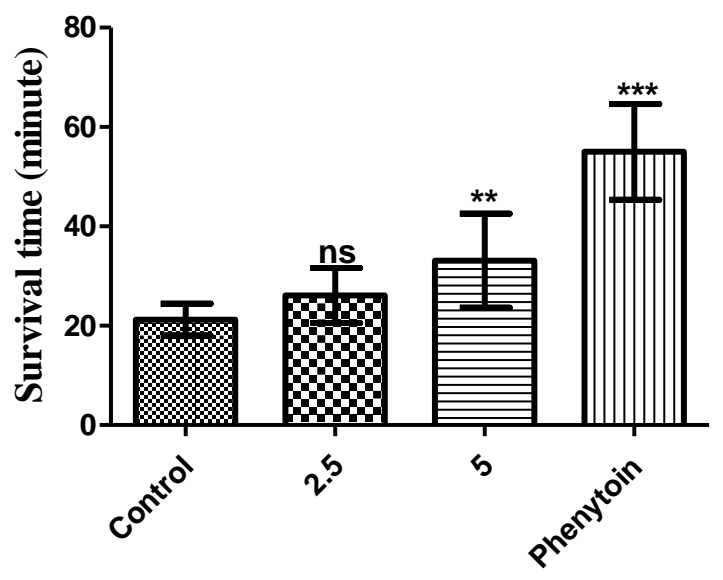

Concentration $(\mathrm{mg} / \mathrm{kg})$

Fig. 1: Antihypoxic activities of edaravone in asphyctic hypoxia in mice. Data are expressed as mean $\pm S D(n=8)$, ( $n$ s, not significant, $* * P<0.01$, ***P<0.001, compared to control).

The results of the circulatory hypoxia are shown in Figure 2 . Edaravone at $5 \mathrm{mg} \mathrm{kg}^{-1}$ was pointedly effective. It significantly prolonged the latency for death with respect to the control group $(28.30 \pm 6.66$ vs. $9.77 \pm 1.16 \mathrm{~min}, P<0.001)$. This effect was dose-dependent. At $2.5 \mathrm{mg}$ $\mathrm{kg}^{-1}$, it also prolonged the survival time $(12.05 \pm 1.55 \mathrm{~min})$, but this effect was not statistically significant from the control $(P>0.05)$.

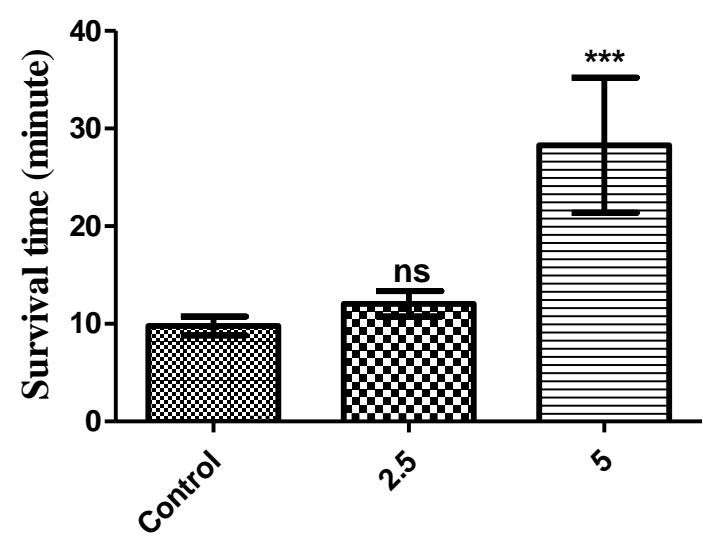

Concentration (mg/kg)

Fig. 2. Antihypoxic activity of edaravone in circulatory hypoxia in mice. Data are expressed as mean $\pm S D(n=8)$, (ns, not significant, $* * * P<0.001$, compared to control $)$. 
Compounds showed good activity in the haemic model (Figure 3). The control group died because of hypoxia in $8.01 \pm 0.83 \mathrm{~min}$. Edaravone at $5 \mathrm{mg} \mathrm{kg}^{-1}$ prolonged the latency for death, but this activity was not statistically significant from the control group $(9.57 \pm 3.33 \mathrm{~min}$, $P>0.05)$. At $10 \mathrm{mg} \mathrm{kg}^{-1}$, edaravone showed statistically significant activity compared to the control group $(P<0.01)$. It significantly prolonged the latency for death with respect to the control group $(13.14 \pm 3.71)$.

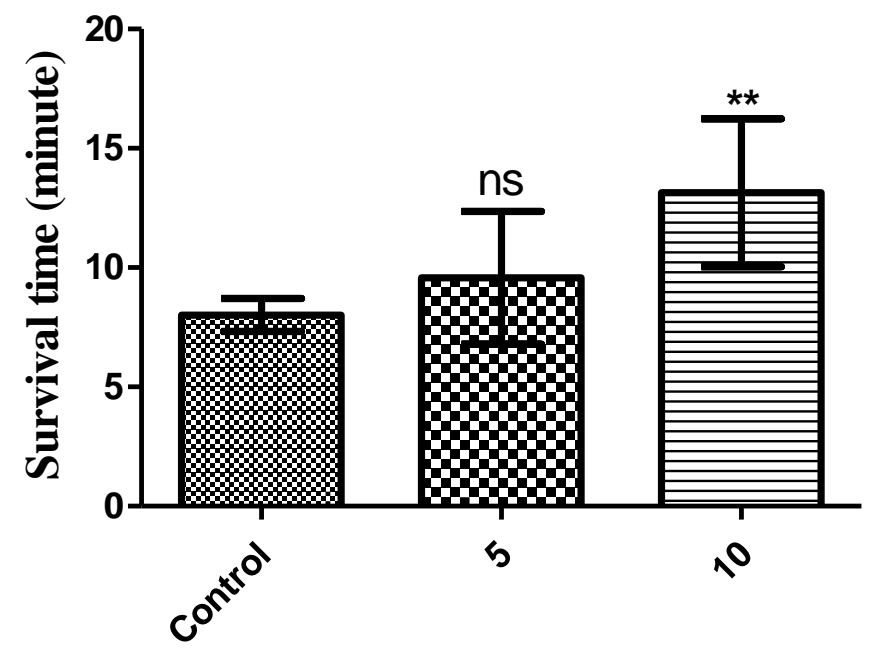

Concentration (mg/kg)

Fig. 3. Antihypoxic activity of edaravone in haemic hypoxia in mice. Data are expressed as mean $\pm S D(n=8),(n s$, not significant, $* * P<0.01$, compared to control).

Elevation of MDA is known as an important marker for oxidative stress. The level of MDA as an indicator of lipid peroxidation in the asphytic hypoxia model is shown in Figure 4.

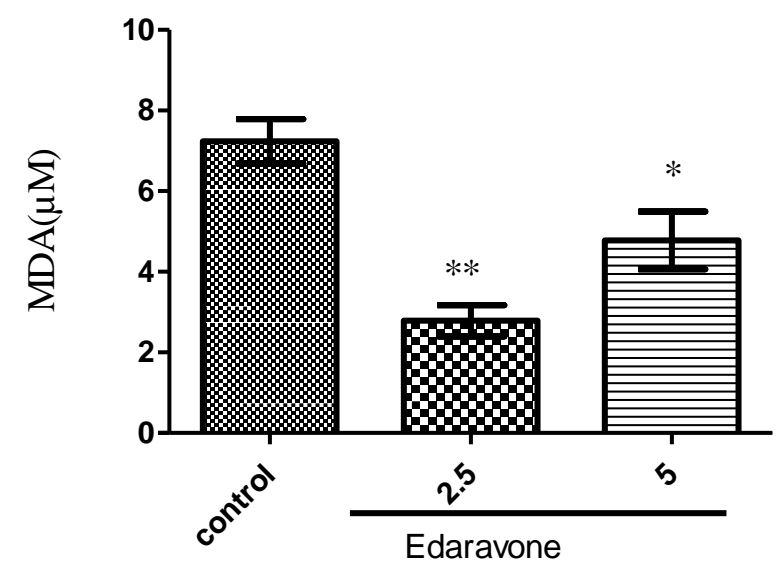

Fig.4. Effects of Edaravone on asphyctic hypoxia-induced lipid peroxidation in brain tissue in mice. Data were expressed as mean $\pm S D(n=6)$. *Significantly different from control group $(P<0.05)$, **Significantly different from control group $(P<0.01)$. 
Edaravone as a dose-dependent drug hugely prevented the asphytic hypoxia-induced LPO with respect to the control $(P<0.05)$. As shown in figure 5, Edaravone at both doses of 2.5 and $5 \mathrm{mg}$ $\mathrm{kg}^{-1}$ decreased lipid peroxidation due to circulatory hypoxia conditions; nonetheless, this effect was not significant. Also, the assessment of MDA concentration in the haemic model showed that edaravone significantly $(P<0.05)$ inhibited the increased lipid peroxidation induced by haemic hypoxia at both doses of 2.5 and $5 \mathrm{mg} \mathrm{kg}^{-1}$ (Fig. 6).

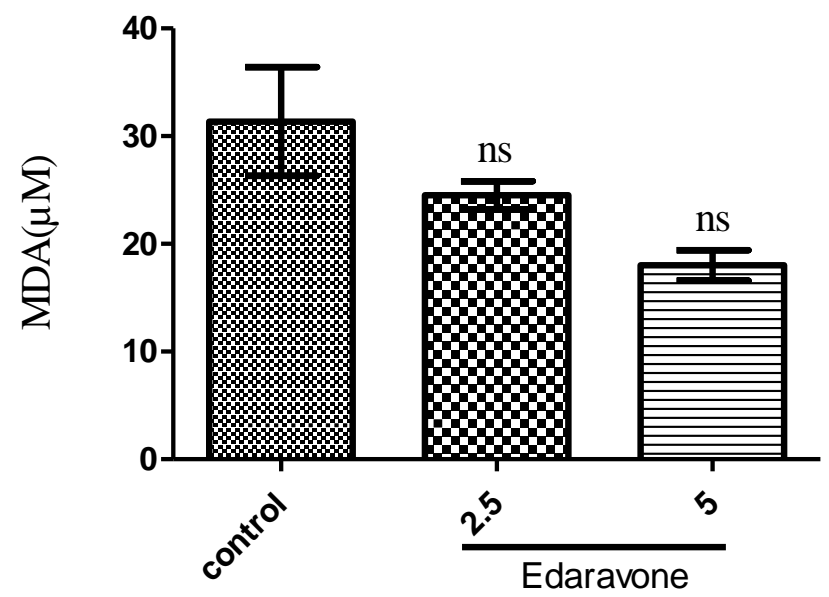

Fig.5. Effects of Edaravone on circulatory hypoxia-induced lipid peroxidation in brain tissue in mice. Data were expressed as mean $\pm S D(n=6)$.

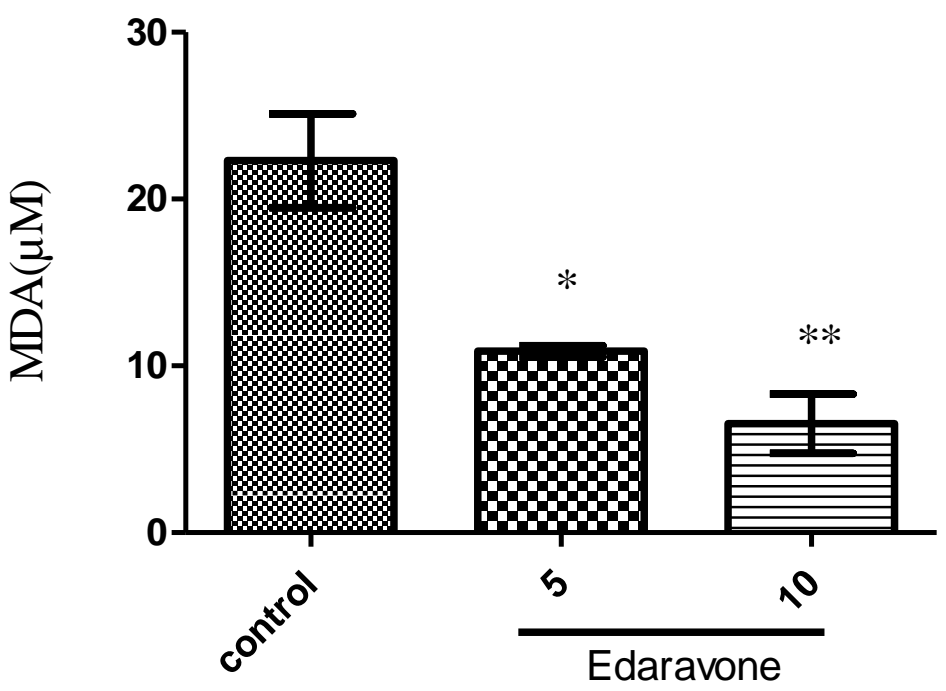

Fig.6. Effects of Edaravone on haemic hypoxia-induced lipid peroxidation in brain tissue in mice. Data were expressed as mean $\pm S D(n=6)$. *Significantly different from control group $(P<0.05)$, ** Significantly different from control group $(P<0.01)$. 
The GSH levels (as the main intracellular antioxidant) in the brain tissue of an asphytic hypoxia mice treated with edaravone $\left(2.5\right.$ and $\left.5 \mathrm{mg} \mathrm{kg}^{-1}\right)$ was significantly $(P<0.05)$ increased in compared with the control group (Fig. 7).

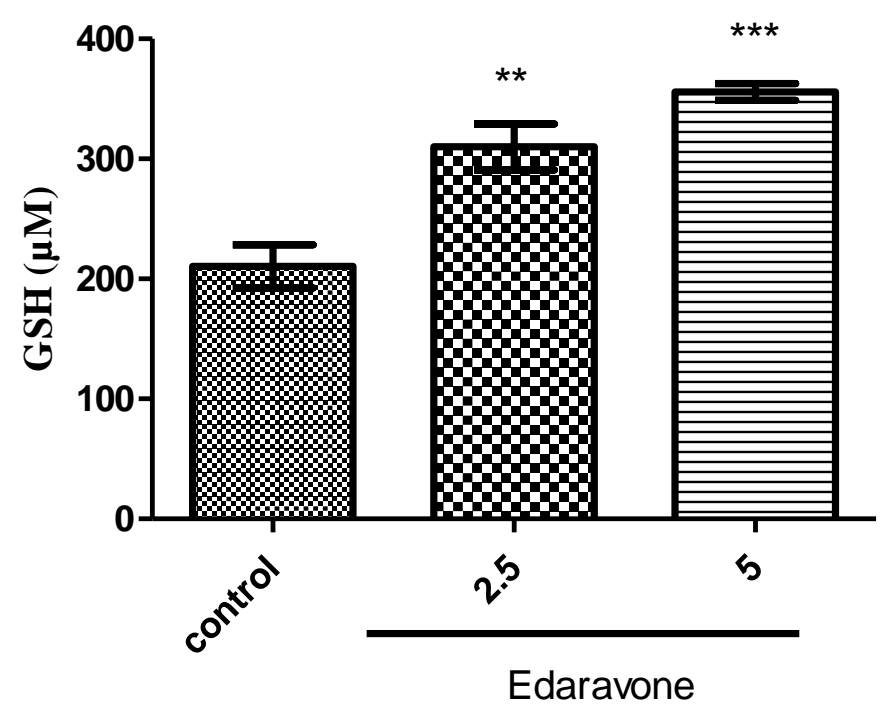

Fig.7. Effects of Edaravone on asphyctic hypoxia-induced GSH oxidation in brain tissue in mice.

Data were expressed as mean $\pm S D(n=6)$. **Significantly different from control group

$$
(P<0.01) \text {, *** Significantly different from control group }(P<0.001) \text {. }
$$

As shown in figure 8 , Edaravone at both doses of 2.5 and $5 \mathrm{mg} \mathrm{kg}^{-1}$ significantly inhibited GSH oxidation due to the circulatory hypoxia conditions $(P<0.05)$. The assessment of MDA concentration in the haemic model showed that edaravone significantly $(P<0.05)$ inhibited the increased lipid peroxidation induced by haemic hypoxia at both doses of 2.5 and $5 \mathrm{mg} \mathrm{kg}^{-1}$ (Fig. 9).

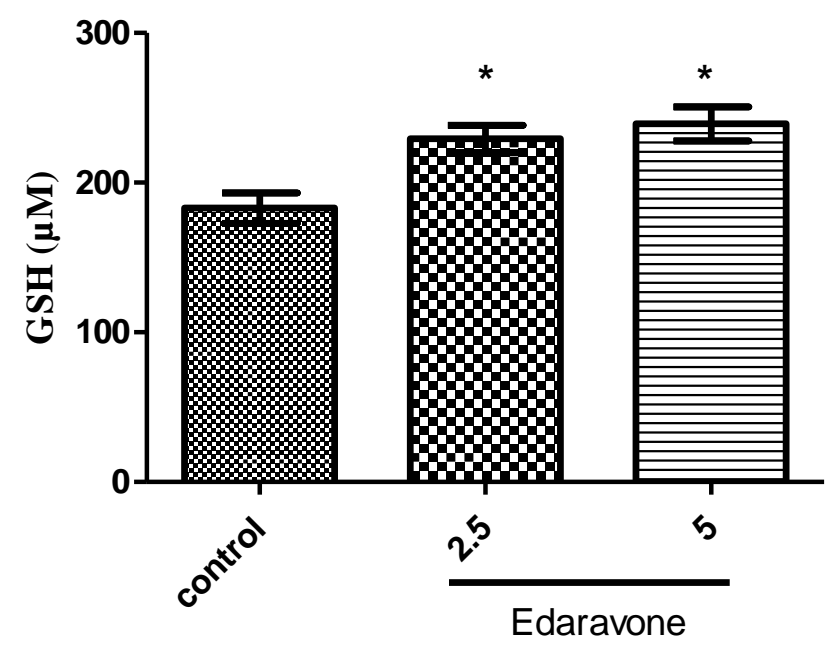

Fig.8. Effects of Edaravone on circulatory hypoxia-induced GSH oxidation in brain tissue in mice. Data were expressed as mean $\pm S D(n=6)$.

*Significantly different from control group $(P<0.05)$. 


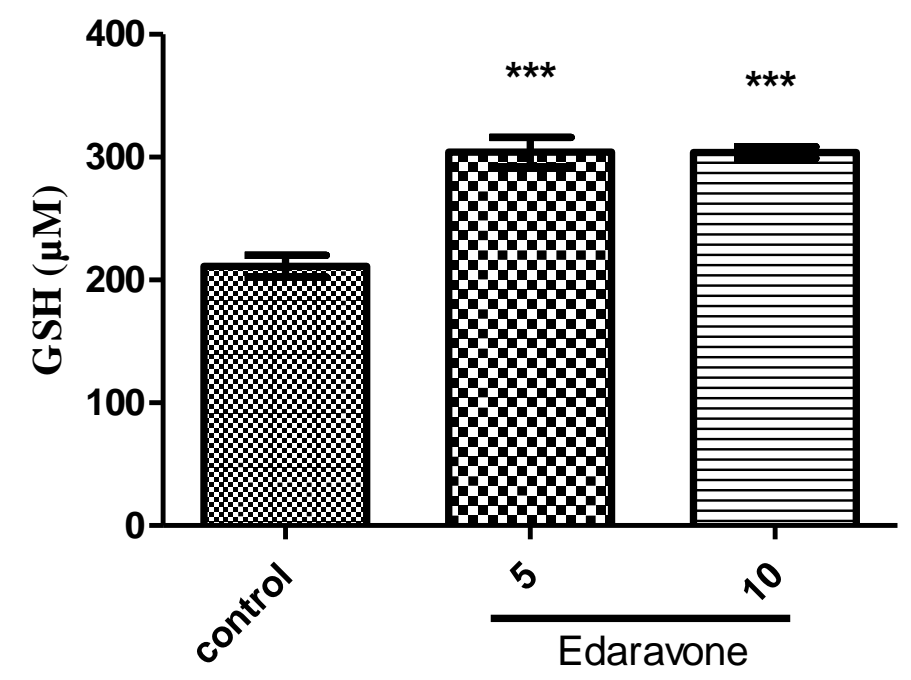

Fig.9. Effects of Edaravone on haemic hypoxia-induced GSH oxidation in brain tissue in mice.

Data were expressed as mean $\pm S D(n=6)$. ***Significantly different from control group $(P<0.001)$.

\section{Discussion}

Hypoxia produces strong physiologic stress and induces a wide range of deleterious effects at the cellular level. Oxygen is a vital element for most organisms and is necessary for normal redox reactions in the cell. Also, oxygen can be a source of ROS formation in cells ${ }^{17}$. It has been shown that both low (hypoxia) and high (hyperoxia) concentration of oxygen can promote oxidative damage and increase the risk of morbidity and mortality ${ }^{18}$. Previous studies showed that hypoxia disturbs mitochondrial function and result in increased ROS production and oxidative stress, which could trigger apoptosis signaling ${ }^{19,20}$.

The brain, which consumes a large quantity of oxygen, is seriously vulnerable to low levels of oxygen ${ }^{21}$. Free radicals act as signaling species in various normal physiological processes, but excessive production of these radicals causes damage to biological material. The increased level of ROS in hypoxia is the result of the accumulation of reducing equivalents in the mitochondrial electron transport system ${ }^{22}$. The effects of ROS can be particularly evident in specific tissues such as the brain since it consumes approximately 1/5 of the basal oxygen. Many efforts have been undertaken to develop therapies to reduce the effects of oxidative stress. Considerable evidence shows that antioxidants can exert protecting action on a variety of illnesses. Polyphenols are potent antioxidants and have a broad spectrum of pharmacological and therapeutic effects ${ }^{23}$.

Statistically significant antihypoxic activities were established in $5 \mathrm{mg} \mathrm{kg}^{-1}$ dose of edaravone in experimental models of hypoxia in mice. At this dose, edaravone showed statistically significant activity with respect to the control group. This effect was dose-dependent. At a higher tested 
dose, this drug showed statistically significant activity with respect to the control group. Protective effects of edaravone have been reported against cerebral ischemia-reperfusion injuries in some experimental animal models ${ }^{5,6}$. A close relationship between oxidative metabolism and cholinergic function has been found during the investigations of $\mathrm{NaNO}_{2}$ on brain metabolism ${ }^{24}$. Chemical hypoxia is induced by the injection of $\mathrm{NaNO}_{2}$, which reduces the oxygen-carrying capacity of the blood by converting hemoglobin to methemoglobin. This lethal dose is injected 30 min after the phenolic treatment. Immediately after the $\mathrm{NaNO}_{2}$ injection, the animals are placed in small cages and the time between injection of $\mathrm{NaNO}_{2}$ and cessation of respiration is recorded. Edaravone showed good activity in haemic model.

Available research studies illustrate that using $\mathrm{NaF}$ induces circulatory hypoxia which increases the blood histamine content and decreases the oxygen-carrying capacity. Edaravone at $5 \mathrm{mg} \mathrm{kg}^{-1}$ was highly effective. The mechanism of this protective action may be due in part to the antioxidant activity of edaravone. Because there is no standard drug for haemic and circulatory hypoxic models, results of this study were compared to those of control groups.

Assessment of oxidative stress markers in the brain tissue of mice in three hypoxia models showed that edaravone reduced the level of oxidative stress in comparison with the control group. In fact, the hypoxia induced the ROS production and can cause oxidation of other cellular macromolecules such as protein ${ }^{25,26}$, DNA, and RNA ${ }^{27,28}$, lipid peroxidation ${ }^{17,29,30}$ and neuronal dysfunction or death. Lipid peroxidation is one of the most critical indicators of oxidative stress that has harmful effects on cell or tissue and can lead to more free radical production via chain reaction, and finally, cell membrane disruption.

The result of the present study revealed that hypoxia causes lipid peroxidation in brain tissue, which significantly inhibited by edaravone. Previous studies showed that edaravone prevented lipid peroxidation and the production of nitric oxide in the neonatal rat brain following hypoxicischemic insult and led to attenuation of neuronal damage in the neonatal rat brain ${ }^{31,32}$. Another study demonstrated that Prophylactic administration of edaravone ameliorated transient hypoxicischemic brain injury via reducing oxidative stress ${ }^{33}$.

Glutathione (GSH) is the primary antioxidant in the cellular system that directly scavenges free radicals ${ }^{34}$. Previous studies showed a fall in GSH level. In the present study, after hypobaric hypoxia that may be due to inhibition of GSH synthesis and increased utilization of GSH for detoxification of hypoxia induced free radical ${ }^{4}$.

Further in the present study, a decreased in GSH concentration has been observed. This leads to an aggravation of oxidative stress level and more brain injury. Treatment with edaravone significantly restored GSH concentration in brain tissue after hypoxia conditions. 


\section{Conclusion}

Edaravone showed an excellent protective effect against hypoxia in all tested models as well as a decrease of oxidative stress in the brain tissue of hypoxic mice. Notably, they produced a significant and dose-dependent effect on the models of asphytic and circulatory hypoxia. The antioxidant activity may be a proposed mechanism for reported antihypoxic activities of this drug.

\section{Conflict of Interest}

None.

\section{Acknowledgments}

The authors express their appreciation to the Vice-Chancellor for Research at Mazandaran University of Medical Sciences for financial support of the current study. 


\section{References}

1. Kiang JG, Tsen K. Biology of hypoxia. Chinese Journal of Physiology. 2006;49(5):223.

2. Shokrzadeh M, Zamani E, Mehrzad M, Norian Y, Shaki F. Protective effects of propofol against Methamphetamine-induced neurotoxicity. Toxicology International. 2015;22(1):92.

3. Hosseini M-J, Shaki FS, Ghazi-Khansari M, Pourahmad J. Toxicity of arsenic (III) on isolated liver mitochondria: a new mechanistic approach. Iranian Journal of Pharmaceutical Research. 2013;12:121-138.

4. Maiti P, Singh SB, Sharma AK, Muthuraju S, Banerjee PK, Ilavazhagan G. Hypobaric hypoxia induces oxidative stress in rat brain. Neurochemistry international. 2006;49(8):709-716.

5. Sinha M, Anuradha H, Juyal R, Shukla R, Garg R, Kar A. Edaravone in acute ischemic stroke, an Indian experience. Neurology asia. 2009;14(1).

6. Inokuchi Y, Imai S, Nakajima Y, et al. Edaravone, a free radical scavenger, protects against retinal damage in vitro and in vivo. Journal of Pharmacology and Experimental Therapeutics. 2009;329(2):687-698.

7. Roh MI, Murakami Y, Thanos A, Vavvas DG, Miller JW. Edaravone, an ROS scavenger, ameliorates photoreceptor cell death after experimental retinal detachment. Investigative ophthalmology \& visual science. 2011;52(6):3825-3831.

8. Toyoda K, Fujii K, Kamouchi M, et al. Free radical scavenger, edaravone, in stroke with internal carotid artery occlusion. Journal of the neurological sciences. 2004;221(1):1117.

9. Inatomi Y, Takita T, Yonehara T, et al. Efficacy of edaravone in cardioembolic stroke. Internal Medicine. 2006;45(5):253-257.

10. Suda S, Igarashi H, Arai Y, Andou J, Chishiki T, Katayama Y. Effect of edaravone, a free radical scavenger, on ischemic cerebral edema assessed by magnetic resonance imaging. Neurologia medico-chirurgica. 2007;47(5):197-202.

11. Watanabe T, Tanaka M, Watanabe K, Takamatsu Y, Tobe A. Research and development of the free radical scavenger edaravone as a neuroprotectant. JOURNALPHARMACEUTICAL SOCIETY OF JAPAN. 2004;124(3):99-112.

12. Song Y, GU Q. Edaravone (MCI-186), a free radical scavenger, attenuates retinal ischemia/reperfusion injury in rats1. Acta Pharmacologica Sinica. 2008;29(7):823-828.

13. Eslami B, Nabavi S, Nabavi S, Ebrahimzadeh M, Mahmoudi M. Pharmacological activities of Hypericum scabrum L. Eur Rev Med Pharmacol Sci. 2011;15:532-537.

14. Ebrahimzadeh MA, Nabavi SF, Nabavi SM, Eslami B. Antihypoxic and antioxidant activity of Hibiscus esculentus seeds. Grasas y aceites. 2010;61(1):30-36.

15. Zhang F, Xu Z, Gao J, Xu B, Deng Y. In vitro effect of manganese chloride exposure on energy metabolism and oxidative damage of mitochondria isolated from rat brain. Environmental Toxicology and Pharmacology. 2008;26(2):232-236.

16. Sadegh C, Schreck RP. The spectroscopic determination of aqueous sulfite using Ellman's reagent. MURJ. 2003;8:39-43.

17. Omrani H, Alipour MR, Mohaddes G. Ghrelin improves antioxidant defense in blood and brain in normobaric hypoxia in adult male rats. Advanced pharmaceutical bulletin. 2015;5(2):283.

18. Zhao HW, Haddad GG. Review: Hypoxic and oxidative stress resistance in Drosophila melanogaster. Placenta. 2011;32:S104-S108. 
19. Guzy RD, Mack MM, Schumacker PT. Mitochondrial complex III is required for hypoxia-induced ROS production and gene transcription in yeast. Antioxidants \& redox signaling. 2007;9(9):1317-1328.

20. Satoh T, Enokido Y, Aoshima H, Uchiyama Y, Hatanaka H. Changes in mitochondrial membrane potential during oxidative stress-induced apoptosis in PC12 cells. Journal of neuroscience research. 1997;50(3):413-420.

21. Warner DS, Sheng H, Batinić-Haberle I. Oxidants, antioxidants and the ischemic brain. Journal of experimental biology. 2004;207(18):3221-3231.

22. Tibor B, Zsolt R. High altitude and free radicals. J Sports Sci Med. 2004;3:64-69.

23. Spencer JP. Beyond antioxidants: the cellular and molecular interactions of flavonoids and how these underpin their actions on the brain. Proceedings of the Nutrition Society. 2010;69(02):244-260.

24. Gibson GE, Shumada M, Blass JP. Alterations in acetylcholine synthesis and cyclic nucleotides in mild cerebral hypoxia. Journal of neurochemistry. 1978;31(4):757-760.

25. Stadtman ER, Berlett BS. Reactive oxygen-mediated protein oxidation in aging and disease. Chemical research in toxicology. 1997;10(5):485-494.

26. Hensley K, Hall N, Subramaniam R, et al. Brain regional correspondence between Alzheimer's disease histopathology and biomarkers of protein oxidation. Journal of neurochemistry. 1995;65(5):2146-2156.

27. Lovell MA, Gabbita SP, Markesbery WR. Increased DNA oxidation and decreased levels of repair products in Alzheimer's disease ventricular CSF. Journal of neurochemistry. 1999;72(2):771-776.

28. Gabbita SP, Lovell MA, Markesbery WR. Increased nuclear DNA oxidation in the brain in Alzheimer's disease. Journal of neurochemistry. 1998;71(5):2034-2040.

29. Butterfield DA, Lauderback CM. Lipid peroxidation and protein oxidation in Alzheimer's disease brain: potential causes and consequences involving amyloid $\beta$ peptide-associated free radical oxidative stress 1, 2. Free Radical Biology and Medicine. 2002;32(11):1050-1060.

30. Markesbery W, Lovell M. Four-hydroxynonenal, a product of lipid peroxidation, is increased in the brain in Alzheimer's disease. Neurobiology of aging. 1998;19(1):33-36.

31. Noor JI, Ikeda T, Ueda Y, Ikenoue T. A free radical scavenger, edaravone, inhibits lipid peroxidation and the production of nitric oxide in hypoxic-ischemic brain damage of neonatal rats. American journal of obstetrics and gynecology. 2005;193(5):1703-1708.

32. Noor JI, Ueda Y, Ikeda T, Ikenoue T. Edaravone inhibits lipid peroxidation in neonatal hypoxic-ischemic rats: an in vivo microdialysis study. Neuroscience letters. 2007;414(1):5-9.

33. Sun Y-Y, Li Y, Wali B, et al. Prophylactic Edaravone Prevents Transient HypoxicIschemic Brain Injury Implications for Perioperative Neuroprotection. Stroke. 2015;46(7):1947-1955.

34. Shaki F, Pourahmad J. Mitochondrial toxicity of depleted uranium: Protection by betaglucan. Iranian Journal of Pharmaceutical Research. 2012;12(1):131-140. 